\title{
Radial sine-Gordon kinks as sources of fast breathers
}

\author{
Caputo, Jean Guy; Sørensen, Mads Peter
}

Published in:

Physical Review E

Link to article, DOI:

10.1103/PhysRevE.88.022915

Publication date:

2013

Document Version

Publisher's PDF, also known as Version of record

Link back to DTU Orbit

Citation (APA):

Caputo, J. G., \& Sørensen, M. P. (2013). Radial sine-Gordon kinks as sources of fast breathers. Physical Review E, 88(2), [022915]. https://doi.org/10.1103/PhysRevE.88.022915

\section{General rights}

Copyright and moral rights for the publications made accessible in the public portal are retained by the authors and/or other copyright owners and it is a condition of accessing publications that users recognise and abide by the legal requirements associated with these rights.

- Users may download and print one copy of any publication from the public portal for the purpose of private study or research.

- You may not further distribute the material or use it for any profit-making activity or commercial gain

- You may freely distribute the URL identifying the publication in the public portal

If you believe that this document breaches copyright please contact us providing details, and we will remove access to the work immediately and investigate your claim. 


\title{
Radial sine-Gordon kinks as sources of fast breathers
}

\author{
J.-G. Caputo ${ }^{1, *}$ and M. P. Soerensen ${ }^{2, \dagger}$ \\ ${ }^{1}$ Department of Mathematics, University of Arizona, Tucson, Arizona 85719, USA \\ ${ }^{2}$ Department of Applied Mathematics and Computer Science, Technical University of Denmark, DK-2800 Kgs. Lyngby, Denmark
}

(Received 18 March 2013; published 19 August 2013)

\begin{abstract}
We consider radial sine-Gordon kinks in two, three, and higher dimensions. A full two-dimensional simulation showing that azimuthal perturbations remain small allows us to reduce the problem to the one-dimensional radial sine-Gordon equation. We solve this equation on an interval $\left[r_{0}, r_{1}\right]$ and absorb all outgoing radiation. As the kink shrinks toward $r_{0}$, before the collision, its motion is well described by a simple law derived from the conservation of energy. In two dimensions for $r_{0} \leqslant 2$, the collision disintegrates the kink into a fast breather, while for $r_{0} \geqslant 4$ we obtain a kink-breather metastable state where breathers are shed at each kink "return." In three and higher dimensions $d$, an additional kink-oscillon state appears for small $r_{0}$. On the application side, the kink disintegration opens the way for new types of terahertz microwave generators.
\end{abstract}

DOI: 10.1103/PhysRevE.88.022915

PACS number(s): 05.45.Yv

\section{INTRODUCTION}

The sine-Gordon equation is an important model both for theory and for applications. It corresponds to a classical field with degenerate ground states $(2 n \pi)$ [1]. In one space dimension, it is integrable via the inverse-scattering transform and it has two main classes of localized solutions, namely the kink and the breather. The former is particularly interesting because it is a topological defect separating two regions where the solution is 0 and $2 \pi$. In higher dimensions, one can introduce the radial kink, i.e., a kink which only depends on the radius $r$, and this was studied by a number of authors. Among these, Christiansen et al. [2-4] have shown that such kinks with initial velocity exhibit the return effect where they "grow" up to some radius and then shrink back. Note also the remarkable work by Geicke, who described solutions of the radial sine-Gordon equation $[5,6]$ and indicated that radial kinks are destroyed [7] at the origin in two dimensions. This was also observed by Bogolubsky and Makhankhov [8]. This particular phenomenon is not well understood. Geicke [7] reports in particular a difference in the collapse of the kink in two and three dimensions. The second class of solutions, the breather, can be generalized to higher dimensions. In two dimensions, some such solutions were observed numerically to be very long lived; for example, Ref. [9] gives initial conditions leading to such solutions. These "pulsons" or "oscillons" can also be found in the sister model of the sine-Gordon equation, i.e., the $\phi^{4}$ equation. For both models, the frequency of the oscillons is below the gap but their long life in two dimensions has just been explained recently [10].

To analyze these radial solutions, one can assume that the radial term is small so that the system is a perturbed one-dimensional sine-Gordon equation. The main assumption is that the width of the kink remains small compared to its radius. Such a perturbation theory based on inverse scattering was formulated by Maslov [11]. The radiation generated by a shrinking sine-Gordon kink was computed by Malomed

\footnotetext{
*On leave from Laboratoire de Mathématiques, INSA de Rouen, France; caputo@insa-rouen.fr

${ }^{\dagger}$ mpso@dtu.dk
}

[12]; for a $\phi^{4}$ kink, it was computed in Ref. [13]. These estimates were never compared systematically to the numerical solutions. On the other hand, when the radius of the solution is small, the perturbation term becomes very large. Then only conservation laws can be used, so the analysis becomes very difficult. For oscillons, Gleiser [10] showed their stability in two dimensions by using a Gaussian ansatz. Alfimov and Vazquez [14] assumed periodicity (which is observed numerically), expanded the solution in Fourier series, and studied the harmonics. They showed that the harmonics could not be bounded in general, so the solution has infinite energy and therefore cannot exist. The only explanation is that the radiation leak exists but is very weak. There is a connection between radial kinks and oscillons because a kink placed at $R_{0}=3$ was shown to give rise to an oscillon [7]. However, a systematic picture of the radial kink collision in two and higher dimensions is missing.

On the application side, the two-dimensional sine-Gordon equation describes the electrodynamics of a Josephson junction between two superconducting films in the absence of external current and dissipation [15]. The wave part comes from Maxwell's equations and the sine nonlinearity from Josephson's constitutive relation. The variable is the phase difference (or flux) $\psi$ between two superconductors. In this context, the kink solution, a "fluxon," carries a flux quantum which generates microwave radiation in the terahertz range when it collides on the boundary of the device. When the lateral geometry of the device is reduced, the fluxon, once created, is "dragged" toward the narrow edge. This suggested a design of a particle detector [16] and also gave rise to the so-called Eiffel junctions with exponential tapered width [17,18]. For these, analysis and a preliminary experimental realization [19] confirmed that no magnetic field is needed to move the kink; current alone suffices. The dynamics was shown to be very regular, contrary to the standard rectangular design. Note also the analysis of the resonances by Jaworski [20].

There is a strong link between this Eiffel design and the radial sine-Gordon model, as we will see below. This link, together with the formal studies and the applications, inspired us to undertake a numerical study of the dynamics of twoand higher-dimensional radial kinks. We relied strongly on numerical studies using a careful procedure. We first solved 
the two-dimensional (2D) sine-Gordon equation for a radial kink and showed that azimuthal perturbations remain small. This justifies the reduction to the radial sine-Gordon equation. We studied this equation numerically for a radial kink initial condition on a finite domain $r_{0}<r<r_{1}$, and absorbed all outgoing radiation. This last point is important because the radiation reflecting from the boundary and coming back into the computational domain can perturb strongly the solution. We varied $r_{0}$ systematically from 10 to 0 to see how the radial term $(d-1) \phi_{r} / r$ in the Laplacian affects the collision. The dimension $d$ is another parameter that we varied. The radial term can be seen as a perturbation of the one-dimensional sineGordon equation, and by changing $r_{0}$ we change the magnitude of this perturbation from small to very large. Changing both $r_{0}$ and $d$ systematically provides a coherent picture of the radial kink dynamics in two and higher dimensions.

Before the collision at $r_{0}$, we find that the 2D and 3D radial kinks are well described by a simple equation for the radius obtained from energy conservation. When collision occurs, the kink is always strongly affected when $r_{0} \leqslant 5$. In two dimensions for $r_{0} \leqslant 2$ it disintegrates into a fast breather rapidly ejected away from $r_{0}$. For larger $r_{0}$, we observe a semistable kink-breather bound state which sheds fast breathers at each "return." In all cases, the kink decays to 0 . Interestingly, in three dimensions for $r_{0}=5$ we recover the total destruction of the kink. For $r_{0} \leqslant 5$, all the kink energy cannot be converted into a single breather because the radial term is too strong to prevent it from escaping. Instead we observe a kink-oscillon bound state that ejects small high-frequency (low-energy) breathers. For $d \geqslant 3$, the collision can yield the three states as shown by a study of the $\left(d, r_{0}\right)$ parameter plane. This scenario explains the differences observed by Geike [7] and other authors for the two- and three-dimensional kink collision. It also opens an avenue for new microwave devices which transform a fluxon (kink) into a large microwave pulse.

The article is organized as follows. In Sec. II we illustrate the collapse of a sine-Gordon kink in two dimensions and show that there are no azimuthal effects. This justifies the reduction to the radial sine-Gordon equation. In Sec. III we recall its conservation laws. The conservation of energy provides a simple model for the shrinking, which we compare to the numerical solution. We examine in detail the radial kink collision in two, three, and higher dimensions in Sec. IV, and we characterize the emission of breathers. We conclude in Sec. V and suggest a design for a terahertz radiation source.

\section{THE COLLAPSE OF A SINE-GORDON KINK IN A 2D SECTOR}

To illustrate the problem that we will consider, we present here a 2D numerical study of the dynamics of a radial kink in a sector. The $2 \mathrm{D}$ sine-Gordon equation reads

$$
\psi_{t t}-\frac{\psi_{\theta \theta}}{r^{2}}-\psi_{r r}-\frac{\psi_{r}}{r}+\sin \psi=0 .
$$

As a domain, we consider the sector $r_{0}<r<r_{1}, 0<\theta<\theta_{0}$ shown in Fig. 1. The boundary conditions corresponding to no external current are homogeneous Neuman so that $\psi_{\theta}=0$ for $\theta=0, \theta_{0}$ and $\psi_{r}=0$ for $r=r_{0}, r_{1}$.

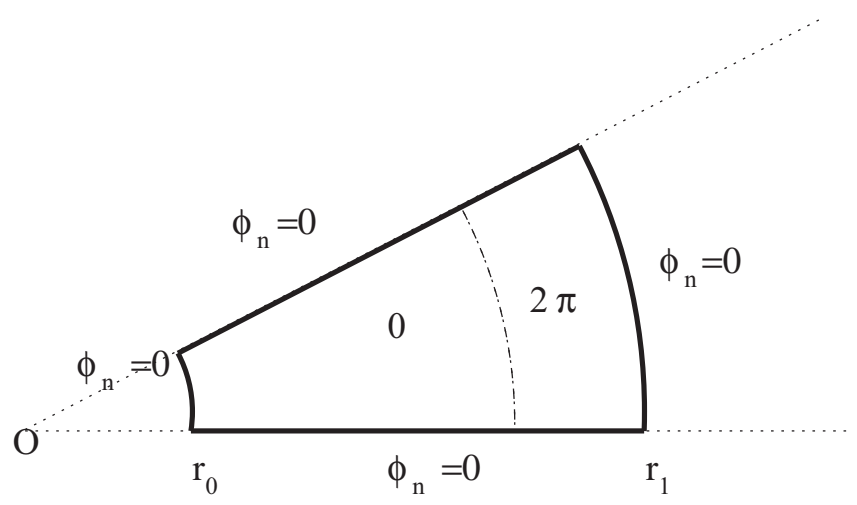

FIG. 1. Sketch of the 2D domain for the sine-Gordon equation. The radial kink initial condition is shown as a dotted-dashed line.

We consider the propagation of a sine-Gordon kink inside such a sector. In that case, the initial condition is given by [1]

$$
\phi(r, t=0)=4 \operatorname{atan}\left(\frac{r-R_{0}}{\sqrt{1-u_{0}^{2}}}\right),
$$

where $R_{0}$ and $u_{0}$ are, respectively, the initial position and the velocity of the kink.

We have computed the evolution of such an initial condition using the COMSOL finite element software [21]. Figure 2 shows two different snapshots of the evolution of a kink in a domain
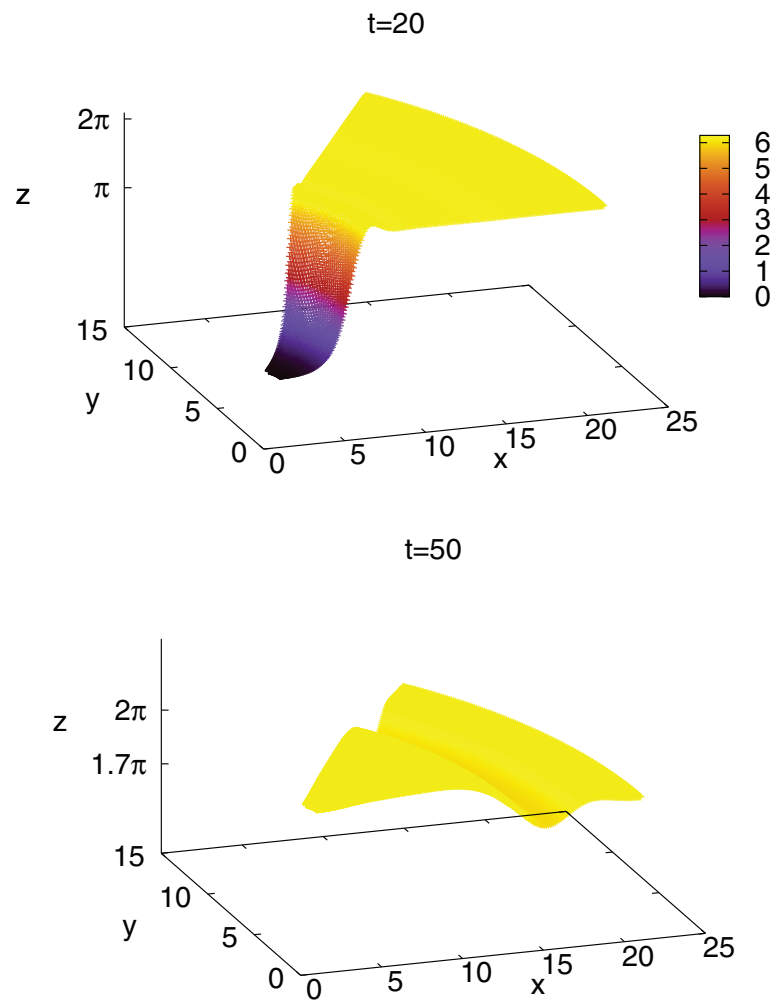

FIG. 2. (Color online) Snapshots of the evolution of a kink for the 2D sine-Gordon equation in a wedge. The kink is started at $R_{0}=15$ in a domain such that $r_{0}=1, r_{1}=20$. The panels show the solution for $t=20$ before the collision and for $t=50$ after the collision. The $z$ color range is $[0 ; 2 \pi]$. 
such that $r_{0}=1, r_{1}=20$ assuming $R_{0}=10$ and $u_{0}=0$. In the top panel, showing the kink at $t=20$, the kink is accelerated toward the narrow edge. Notice the absence of radiation and the characteristic overshoot. The bottom panel presents the solution after collision $t=50$ and shows that the kink has disappeared and only a flat background $\phi \approx 2 \pi$ persists with some oscillations. Despite the violence of the collision, all the energy remains in the radial $n=0$ mode and no azimuthal modes are excited. The space discretization (finite elements) does not preserve the radial symmetry. Nevertheless, azimuthal perturbations do not grow. We therefore now look for a reduction of the model to the radial case and justify this approximation.

To reduce the 2D problem, it is natural to expand in azimuthal modes using the cosine Fourier series,

$$
\psi(r, \theta, t)=\sum_{n=0}^{\infty} \phi_{n}(r, t) \chi_{n}(\theta),
$$

where $\chi_{n}(\theta)=\cos \left(\frac{n \pi \theta}{\theta_{0}}\right)$. Plugging the expression (3) into (1) and projecting onto $\chi_{0}$, we obtain the evolution of $\phi_{0}$,

$$
\begin{aligned}
& -\phi_{0_{t t}}+\phi_{0_{r r}}+\frac{\phi_{0 r}}{r} \\
& =\frac{1}{\theta_{0}} \int_{0}^{\theta_{0}} \sin \left(\phi_{0}+\phi_{1} \chi_{1}+\phi_{2} \chi_{2}+\cdots\right) d \theta .
\end{aligned}
$$

The integrand on the right-hand side can be written as

$$
\begin{aligned}
\sin \left(\phi_{0}+\phi_{1} \chi_{1}+\cdots\right)= & \sin \left(\phi_{0}\right) \cos \left(\phi_{1} \chi_{1}+\phi_{2} \chi_{2}+\cdots\right) \\
& +\cos \left(\phi_{0}\right) \sin \left(\phi_{1} \chi_{1}+\phi_{2} \chi_{2}+\cdots\right) .
\end{aligned}
$$

The integral on the right-hand side of (4) becomes

$$
\begin{aligned}
& \frac{\sin \left(\phi_{0}\right)}{\theta_{0}} \int_{0}^{\theta_{0}} d \theta \cos \left(\phi_{1} \chi_{1}+\phi_{2} \chi_{2}+\cdots\right) \\
& +\frac{\cos \left(\phi_{0}\right)}{\theta_{0}} \int_{0}^{\theta_{0}} d \theta \sin \left(\phi_{1} \chi_{1}+\phi_{2} \chi_{2}+\cdots\right) .
\end{aligned}
$$

To estimate these terms, we expand the cosine and sine. Then we find that the nonzero contribution for the first term will yield terms of the form

$$
\frac{\phi_{i}^{2}}{2} \sin \left(\phi_{0}\right),
$$

and will yield cubic terms for the second integral. This shows that if the $\phi_{i}$ are small, one can assume that

$$
\begin{aligned}
& \sin \left(\phi_{0}+\phi_{1} \chi_{1}+\cdots\right) \\
& \quad \approx \sin \left(\phi_{0}\right)+\cos \left(\phi_{0}\right)\left(\phi_{1} \chi_{1}+\phi_{2} \chi_{2}+\cdots\right),
\end{aligned}
$$

so that (4) reduces to the radial $1 \mathrm{D}$ sine-Gordon equation,

$$
-\phi_{t t}+\phi_{r r}+\frac{\phi_{r}}{r}=\sin (\phi),
$$

where the 0's have been omitted for simplicity. The model (5) can be obtained for any angle $\theta_{0}$, and in particular for the whole two-dimensional sector. It is also linked to the variable width sine-Gordon equation which contains the term $\phi_{x} w^{\prime}(x) / w(x)$ [17]. The radial sine-Gordon equation corresponds to $w(x)=$ $\theta_{0} x$ while the Eiffel junction is for $w(x)=w_{0} e^{-\lambda x}$.

\section{NUMERICAL PROCEDURE}

We now detail the numerical procedure because it is the basis of this work. Another reason is that the approximate analysis based on perturbation methods is difficult to validate a priori for small radii. We give it meaning by comparing the predictions to the numerical solution. We solve the radial sine-Gordon equation using the method of lines where the space discretization is done using a finite difference and the time advance is done using an ode solver (DOPRI5 ordinary differential equation solver [22]). This method is flexible and one can increase easily the space discretization. Another time integrator we have used for comparison is the Verlet method [23]. The number of discretization points for a typical run is 4000 and the accuracy is checked by computing the Hamiltonian $H$ (10). For all cases presented, the relative error is smaller than $2 \times 10^{-5}$. The boundary condition at $r_{0}$ is of the Neuman type so that there is perfect reflection. When $r_{0}=0$, care must be taken because the operator $\phi_{r} / r$ should be regularized because we have an indetermination $0 / 0$. The way to do this is to invoke the limit

$$
\frac{\phi_{r}}{r}=\frac{\phi_{r}(r, t)-0}{r-0} \rightarrow_{r \rightarrow 0} \phi_{r r}(r=0, t),
$$

so that $\phi_{r r}+\phi_{r} /\left.r\right|_{r=0}=\left.2 \phi_{r r}\right|_{r=0}$.

At the instant of collision, radiation is emitted from the kink. To avoid it reentering the computational domain, we introduce a "sponge layer" where waves are damped so that the equation becomes

$$
\phi_{t t}-\phi_{r r}-(d-1) \frac{\phi_{r}}{r}+\sin (\phi)=-\alpha(r) \phi_{t},
$$

where $\alpha(r)$ increases smoothly from $r_{d}=0.9 r_{1}$ to the edge of the domain $r_{1}$ as shown in Fig. 3. This mechanism kills all radiation that travels to the right and exits the computational domain. The amount of energy leaving the computational domain is then computed using the flux relation (12) evaluated at $r_{f}<r_{d}$. This "sponge layer" is better adapted for our purposes than a perfectly patched layer because it damps all outgoing waves, not only the ones of speed one.

Equation (5) is not integrable as in the 1D case, and there are only a finite number of conservation laws. These are the main analytical tool to study the solution. We consider them in the next section.

\section{CONSERVATION LAWS}

The radial sine-Gordon equation in $d$ dimensions,

$$
\phi_{t t}-\phi_{r r}-(d-1) \frac{\phi_{r}}{r}+\sin (\phi)=0
$$

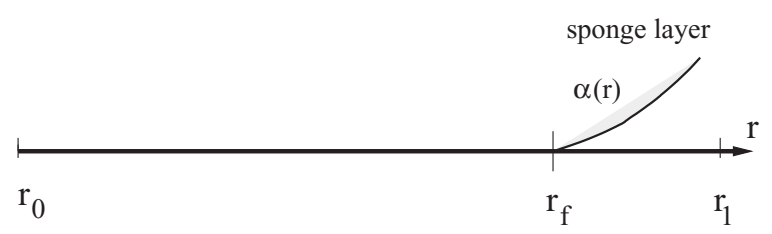

FIG. 3. Sketch of the 1D computational domain. 
in a finite domain $\left[r_{0}, r_{1}\right]$ possesses the following energy conservation law:

$$
\frac{d H}{d t}=\left[r^{d-1} \phi_{r} \phi_{t}\right]_{r_{0}}^{r_{1}},
$$

where the Hamiltonian $H$ is

$$
\begin{aligned}
H & =\int_{r_{0}}^{r_{1}} r^{d-1} d r\left[\frac{\phi_{t}^{2}}{2}+\frac{\phi_{r}^{2}}{2}+(1-\cos \phi)\right] \\
& \equiv \int_{r_{0}}^{r_{1}} r^{d-1} d r \mathcal{H} .
\end{aligned}
$$

To see this, multiply (8) by $\phi_{t}$, integrate over the domain, and integrate by parts the $\phi_{r}$ term. Assuming a Neuman boundary condition at $r=r_{0}$, we naturally obtain the flux relation for the energy,

$$
\frac{d H}{d t}=\left.r_{1}^{d-1} \phi_{r} \phi_{t}\right|_{r_{1}} .
$$

By integrating this relation over time, we obtain

$$
H(t)=H(0)+r_{1}^{d-1} \int_{0}^{t} \phi_{r}\left(r_{1}, t^{\prime}\right) \phi_{t}\left(r_{1}, t^{\prime}\right) d t^{\prime} .
$$

This enables us to compute how much energy leaves the computational domain at $r=r_{f}$. The energy conservation will be crucial to explain many properties of the solution.

Another conservation law is related to the momentum $\Pi$ of the wave defined as

$$
\Pi=\int_{r_{0}}^{r_{1}} r^{d-1} d r \phi_{t} .
$$

From the partial differential equation (8), we get

$$
\frac{d \Pi}{d t}=\left[r^{d-1} \phi_{r}\right]_{r_{0}}^{r_{1}}-\int_{r_{0}}^{r_{1}} r^{d-1} d r \sin \phi,
$$

which shows that even for Neuman boundary conditions at $r=r_{0}, r_{1}$ the momentum is not conserved.

For a localized wave such as a kink, the integrands in $H$ and $\Pi$ are highly localized in $r$. With the Hamiltonian we can then get a good approximation of the motion before collision by using the kink solution to the $1 \mathrm{D}$ sine-Gordon equation as an ansatz,

$$
\phi_{k}=4 \operatorname{atan}\left[\exp \left(\frac{r-R}{\sqrt{(}\left(1-\dot{R}^{2}\right)}\right)\right],
$$

where $R$ is the kink position. Samuelsen [24] used this argument to obtain the kink motion; here we give more details and compare the prediction to the numerical solution. We assume that the kink is not too close to the boundary so the integral can be taken from $-\infty$ to $\infty$. To calculate it, we change the integration variable so that $r-R=r^{\prime}$ and write

$$
H=R \int_{-\infty}^{+\infty} \mathcal{H} d r^{\prime}+\int_{-\infty}^{+\infty} r^{\prime} d r^{\prime} \mathcal{H} .
$$

The second integral is then 0 because of parity. This gives

$$
H \approx 8 \frac{R}{\sqrt{1-(\dot{R})^{2}}} .
$$

At $t=0$, we start the kink at $R=R_{0}$ with $u_{0}$ initial velocity so that $H=8 R_{0}$. Solving the resulting ordinary differential
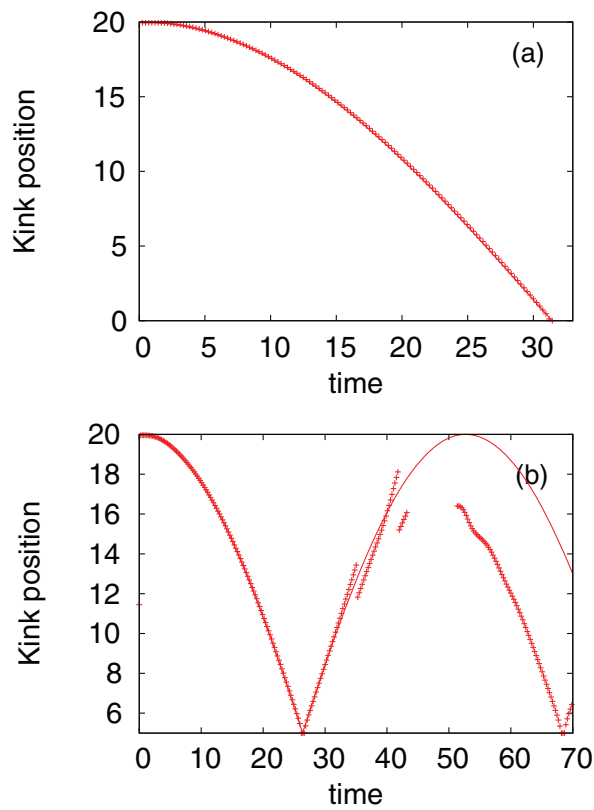

FIG. 4. (Color online) Two-dimensional radial kink position as a function of time for $r_{0}=0$ (a) and $r_{0}=5$ (b). For both plots, the numerical solution is drawn with crosses and the analytical estimates (18)-(20) are shown by the full line.

equation

$$
\frac{d R}{d t}=\sqrt{1-\frac{R^{2}}{R_{0}^{2}}}
$$

we recover the expression of Samuelsen [24],

$$
R=R_{0} \cos \frac{t}{R_{0}}
$$

Note that using the second-order differential equation would give a wrong result. For a minimal radius $r_{0}>0$, the expression can be generalized to

$$
\begin{gathered}
R=R_{0} \cos \frac{t}{R_{0}}, \quad t<t_{0}, \\
R=R_{0} \cos \frac{2 t_{0}-t}{R_{0}}, \quad t>t_{0},
\end{gathered}
$$

where $t_{0}=R_{0} \cos \left(r_{0} / R_{0}\right)$. Figure 4 shows the evolution of the kink position $R(t)$ for the numerical solution, calculated from the maximum of $\phi_{t}$ together with the estimate (18) for $r_{0}=0$ in panel (a). The agreement is very good even for $R$ close to $r_{0}$. The bottom panel shows $R(t)$ for $r_{0}=5$. The estimates (19) and (20) give a good agreement up to $t=33$. After that, radiation is shed from the kink and travels away. Then the estimate breaks down. We come back to this point in the next section.

Using similar arguments, we can reduce the Hamiltonian for the $3 \mathrm{D}$ kink to

$$
H=R^{2} \int_{-\infty}^{+\infty} \mathcal{H} d r^{\prime}+\int_{-\infty}^{+\infty} r^{\prime 2} d r^{\prime} \mathcal{H} .
$$




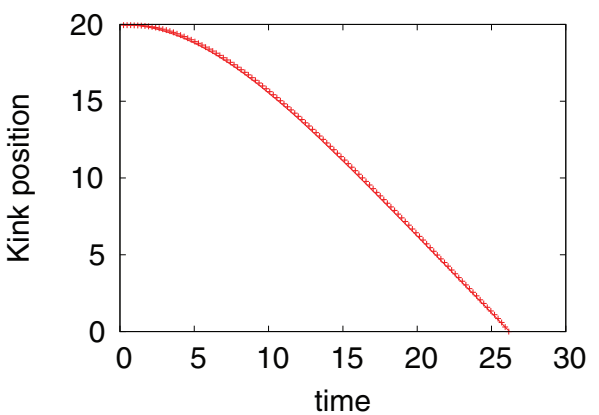

FIG. 5. (Color online) Time evolution of the 3D radial kink position for $r_{0}=0$. The numerical solution is drawn with crosses and the analytical estimate (23) is shown by the full line.

The first term gives

$$
H \approx 8 \frac{R^{2}}{\sqrt{1-(\dot{R})^{2}}}
$$

The second term is a small correction of the order of the cube of the "width" of the kink. It is much smaller than the leading term (21). We then obtain the evolution of the 3D radial kink as

$$
\frac{d R}{d t}=\sqrt{1-\frac{R^{4}}{R_{0}^{4}}}
$$

whose solution is [24]

$$
R=R_{0} \mathrm{cn}\left(\sqrt{2} \frac{t}{R_{0}}, \frac{1}{2}\right)
$$

where $\operatorname{cn}(x, m)$ is the cosine elliptic function [25]. The comparison of (23) with the numerical solution is also good, as shown in the bottom panel of Fig. 5.

\section{RADIAL KINK COLLISION IN TWO AND THREE DIMENSIONS}

To understand the collision of a kink with the boundary at $r=r_{0}$, we have conducted extensive numerical studies varying systematically $r_{0}$. The main result for both two, three, and larger dimensions is that the kink does not survive collision in a proper way when $r_{0}$ is small. It decays after a few collisions, and at each collision it emits part of its energy in the form of fast breathers that escape the radial potential. This is true for all initial conditions $R_{0}>5$. This is interesting since it is a way to destroy the kink. The specifics vary from the $2 \mathrm{D}$ case to the $3 \mathrm{D}$ case, so we will consider them separately.

\section{A. Collision in two dimensions}

Figure 6 shows snapshots of $\phi(x, t)$ as a function of $x$ for different times before and after the collision at $r_{0}=0$. The top panel shows the times $t=11, \ldots, 36$ corresponding to the kink being accelerated toward $r_{0}=0$. Before collision,
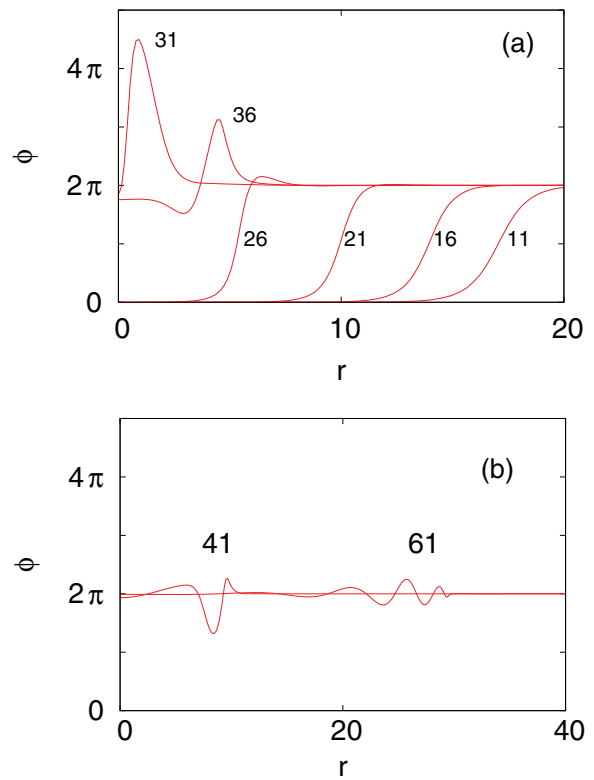

FIG. 6. (Color online) Two-dimensional radial kink collision for $r_{0}=0$. Plot of $\phi(x, t)$ as a function of $x$ for $t=11,16,21,26,31$, and 36 (a) and $t=41$ and 61 (b). The kink is started at $R_{0}=20$. The parameters are $r_{0}=0, r_{1}=40$.

radiation is formed as shown by the "bump" to the left of the solution for $t=26$ [12]. This radiation, however, remains with the kink and grows. This explains why the estimate (18) gives such a good agreement before collision. At collision, the radiation "bump" gives rise to the large overshoot for $t=31$. The panel (b) of Fig. 6 shows the two instants $t=41$ and 61 showing that very little is left of the initial kink. There is just a small disturbance around $2 \pi$ traveling toward large $r$. In fact, all the kink energy from (16) $H \approx 20 \times 8=160$ leaves the computational domain as shown by the energy flux (12) measured at $r=30$ shown in Fig. 7 .

The wave present in the domain after the collision is a fast breather. Recall that a sine-Gordon breather is given by

$$
\phi_{b}=4 \operatorname{atan}\left[\frac{\sqrt{1-\omega^{2}} \cos \left[\omega \gamma\left(t-u r-t_{0}\right)\right]}{\omega \cosh \left[\sqrt{1-\omega^{2}} \gamma\left(r-R_{0}-u t\right)\right]}\right] \text {, }
$$

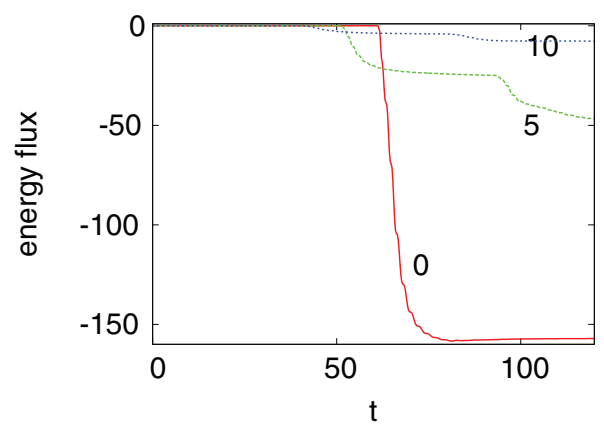

FIG. 7. (Color online) Two-dimensional radial kink collision. Energy exiting the domain at $r_{f}=30$ as a function of time for $r_{0}=0$, 5 , and 10 . The kink is started at the same position $R_{0}=20$ so the initial energy $H_{0} \approx 160$ is the same for the three cases. 
where $\gamma$ is the usual Lorentz factor

$$
\gamma=\frac{1}{\sqrt{1-u^{2}}} .
$$

The energy of the breather on the infinite line is given by [26]

$$
H=16 \gamma \sqrt{1-\omega^{2}},
$$

so that using the same argument as for the kink, we get for the radial case

$$
H_{b}=16 R \gamma \sqrt{1-\omega^{2}}
$$

where $R$ is the center of mass of the breather. To identify this fast breather in the numerical solution, we have plotted the
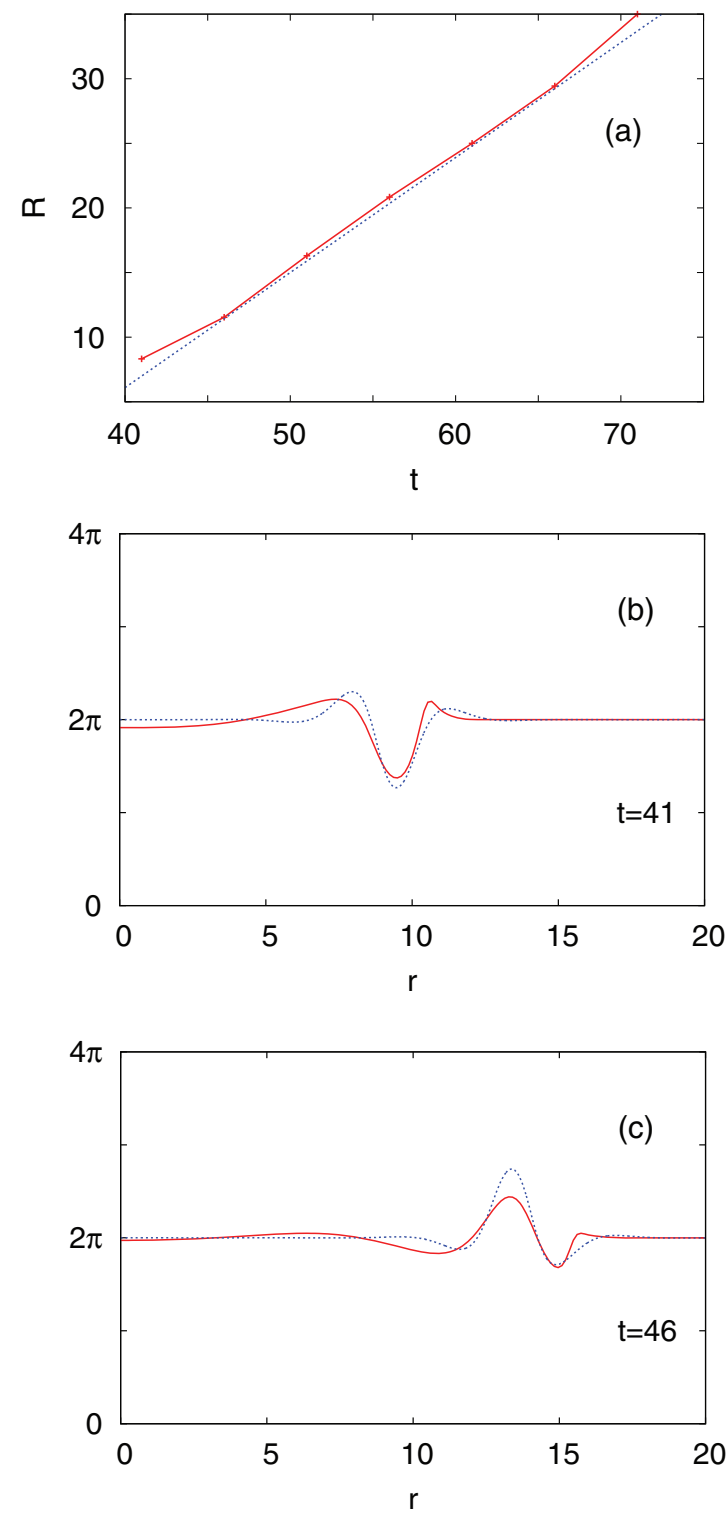

FIG. 8. (Color online) Characterization of the wave after the 2D radial kink collision. The plot (a) shows the center of mass of the wave as a function of time, the numerical solution is shown by the full line (red online), while the fit is shown by the dashed line (blue online). Parts (b) and (c) show the numerical solution by the full line (red online) together with the breather fit (24) by the dashed line (blue online) for $t=41$ and 46 , respectively.
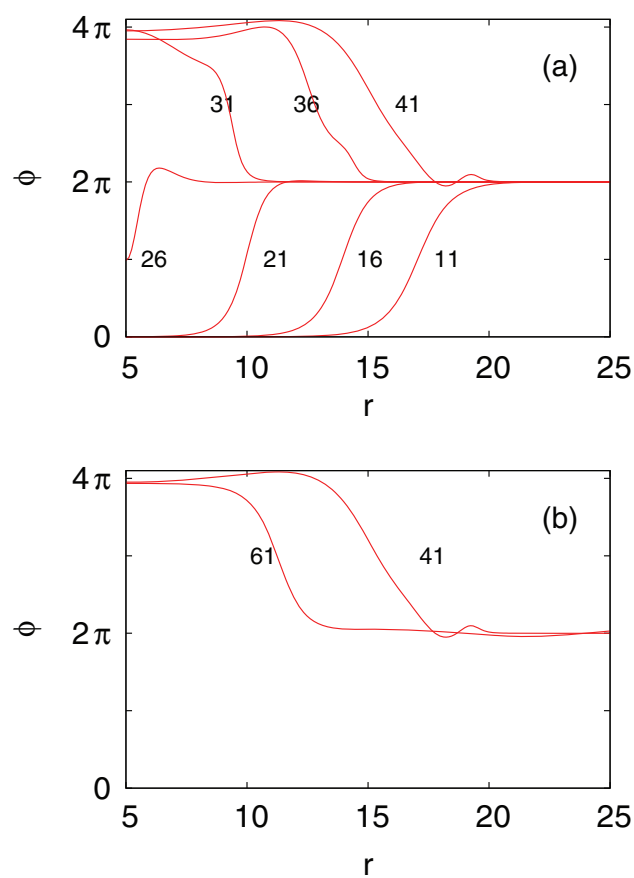

FIG. 9. (Color online) Two-dimensional radial kink collision for $r_{0}=5$. Plots of $\phi(x, t)$ as a function of $x$ for $t=11,16,21,26,31$, 36 , and 41 (a) and $t=41$ and 61 (b). The kink is started at $R_{0}=20$. The parameters are $r_{0}=5, r_{1}=40$.

position of its center of mass as a function of time on the top panel of Fig. 8. The velocity estimated by the fit (dashed line) is $u \approx 0.89$. In the middle and bottom panels, we have plotted
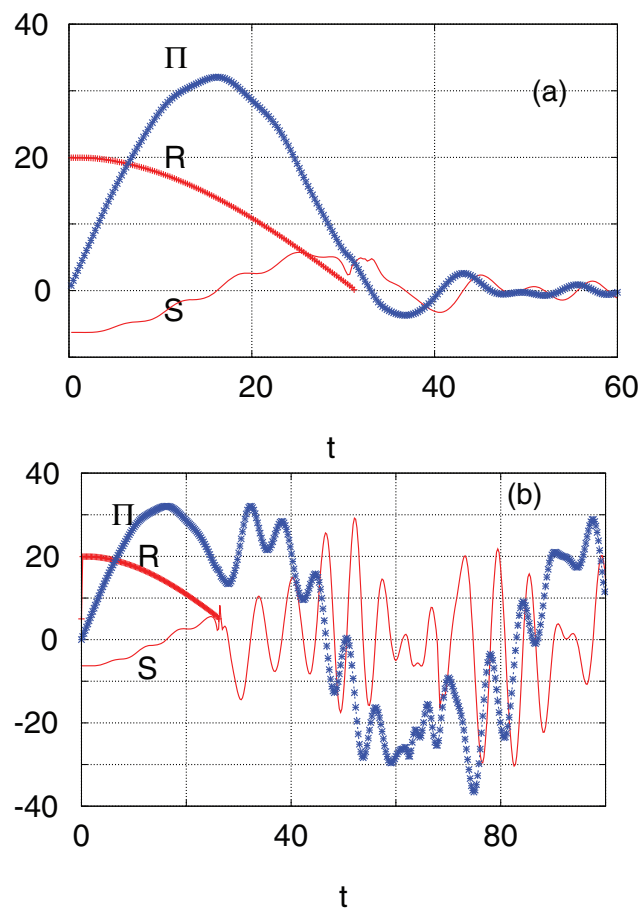

FIG. 10. (Color online) Two-dimensional radial kink collision. Time evolution of the rescaled momentum $\Pi / 2$, the kink position $R$, and the source term $S$ of the momentum equation for $r_{0}=0$ (a) and $r_{0}=5(\mathrm{~b})$. 

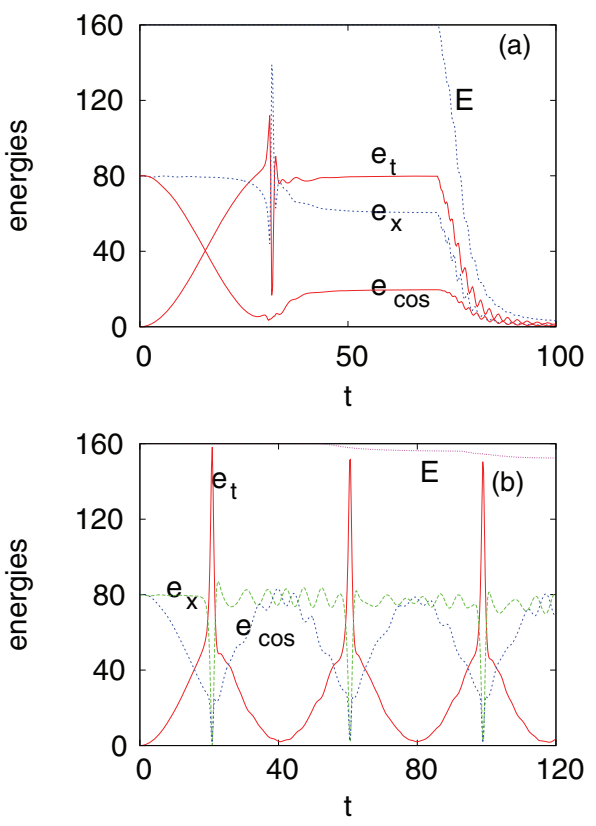

FIG. 11. (Color online) Two-dimensional radial kink collision: Time evolution of the energy components $e_{t}=\int_{r_{0}}^{r_{1}} 0.5 x \phi_{t}^{2} d x, e_{x}=$ $\int_{r_{0}}^{r_{1}} 0.5 x \phi_{x}^{2} d x$, and $e_{\cos }=\int_{r_{0}}^{r_{1}}(1-\cos \phi) x d x$ for a kink. In (a), $r_{0}=$ 0 , while in (b), $r_{0}=10$. The kink is started at the same position, $R_{0}=20$.

the analytical expression of the breather (24) added to the $2 \pi$ background together with the numerical solution for $t=41$ and 46. The parameters used for the fitting are

$$
\omega=0.82, \quad t_{0}=-3, \quad x_{0}=9.2 \text {. }
$$

As can be seen, the fit is very good. The error in the energy between the fit and the numerical solution is $10 \%$. Such a breather can "escape" the radial trap because its high frequency averages out the radial force. Also note that the radial wave equation does not support any traveling wave as in the 1D case, so any emitted radiation has to be in the form of a wave packet. It is interesting to find breather solutions as a product of the disintegration of a kink. In the 1D case for which the sineGordon equation is integrable, the breather and kink-antikink pairs are separated. Here, with the radial term, a connection has been opened between the two states. This is similar to the

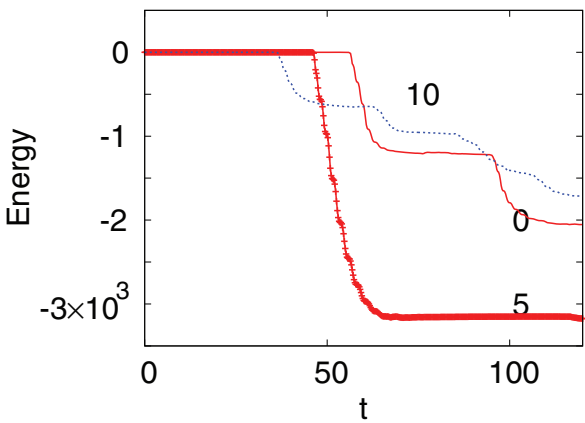

FIG. 12. (Color online) Energy exiting the computational domain as a function of time for three different values $r_{0}=0,5$, and 10 . The kink is started at the same position $R_{0}=20$ so the initial energy $H_{0} \approx 3200$ is the same for the three cases.

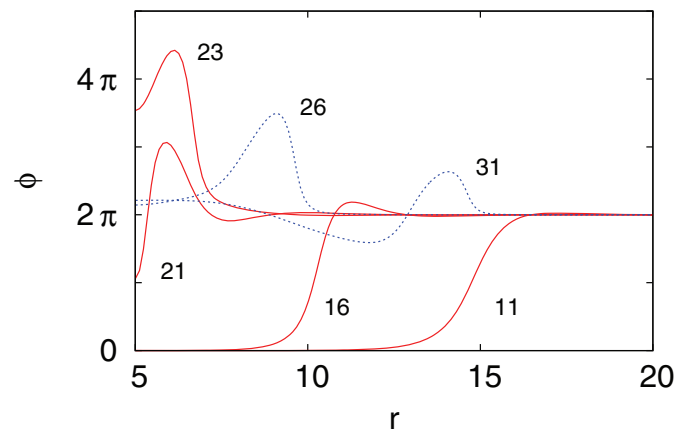

FIG. 13. (Color online) 3D radial kink collision for $r_{0}=5$ : plot of $\phi(x, t)$ as a function of $x$ for $t=11,16,21,23,26$, and 31 . The snapshots after the collision are indicated by the dashed (blue) line. The kink is started at $R_{0}=20$. The parameters are $r_{0}=5, r_{1}=40$.

numerical experiments of [27], where kink-antikink pairs are created out of a train of small breathers in the $\phi^{4}$ model, which is a perturbation of the sine-Gordon equation.

For a larger value of the boundary $r_{0}=5$, the kink is reflected and looks roughly like an antikink. The snapshots are shown in Fig. 9. Notice the return occurring for $t \approx 41$. There is, however, about $20 \%$ of energy (about 21) lost after the collision, as shown in the flux plot Fig. 7. The approximate antikink that is formed has an energy which is about $16 \times 8=128$ so that it "stops" at $R \approx 16$, as shown in the bottom panel of Fig. 9. Again a breather is emitted; it is bound to the antikink up to $t=41$, after which it detaches and propagates to the right. To characterize this breather, we ran the simulation over a much larger space interval, $r_{1}=100$. The parameters of the breather can be calculated as follows using the energy loss:

$$
H(t=0)-H(t=81) \approx 21=x_{0} 16 \frac{\sqrt{1-\omega^{2}}}{\sqrt{1-u^{2}}}
$$

with $x_{0}=30$ corresponding to an instant of observation $t=$ 80. This gives the following parameters:

$$
u=0.7, \quad \omega=0.9995 \text {, }
$$

which are consistent with the energy loss (21) and the duration of the breather passing through the

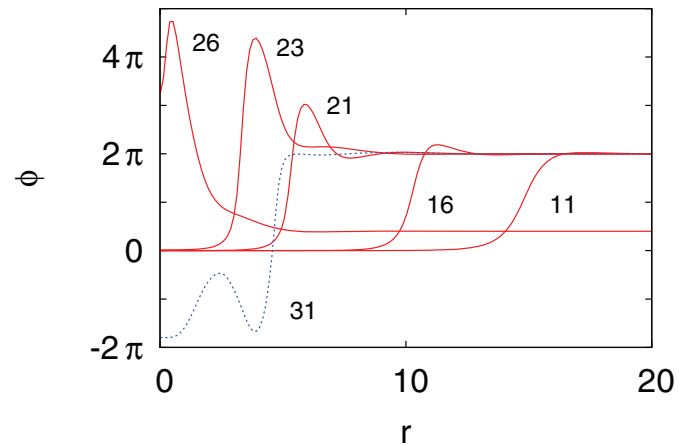

FIG. 14. (Color online) 3D radial kink collision for $r_{0}=0$ : plot of $\phi(x, t)$ as a function of $x$ for $t=11,16,21,23,26$, and 31 . The field for the snapshot $t=23$ has been divided by 5 to fit in the plot. The kink is started at $R_{0}=20$. The parameters are $r_{0}=0, r_{1}=40$. 


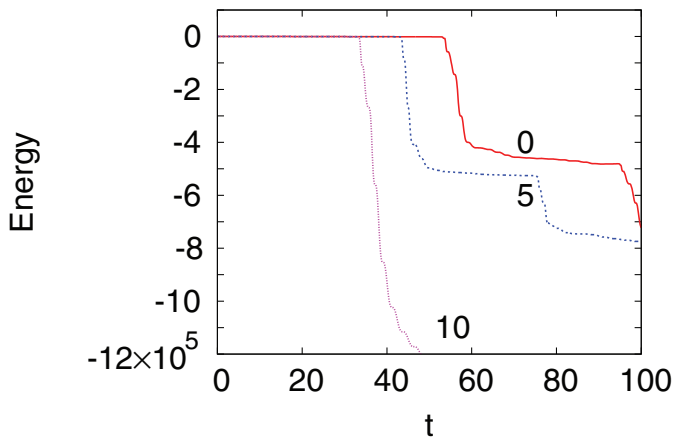

FIG. 15. (Color online) Energy exiting the computational domain [ $\left.r_{0}: 100\right]$ for $d=5$. The curves correspond to $r_{0}=0$ in continuous (red) line, $r_{0}=5$ in dashed (blue) line and $r_{0}=10$ in dotted (pink) line.

boundary,

$$
\delta t=\text { width } / \text { speed }=\frac{\sqrt{1-\omega^{2}}}{\gamma u} \approx 32 .
$$

After the emission of this breather, the kink continues to oscillate and decays slowly, emitting waves at each collision with $r=r_{0}$. For larger $r_{0}$, as shown in Fig. 7 for $r_{0}=10$, the kink decays much slower. For $t=100$, its energy has diminished by about $10 \%$. At every collision some energy is radiated away. For such a value $r_{0}$, the radial term $\phi_{r} / r$ is small and we are close to the one-dimensional situation.

To shed more light on the problem, it is useful to examine the different conservation laws before and after collision. We analyzed the difference between $r_{0}=0$ and 5 by computing the momentum $\Pi$, the right-hand side

$$
-S=-\int_{r_{0}}^{r_{1}} r^{d-1} d r \sin \phi
$$

in the flux of the momentum (14), and the front position $R$. The latter is defined as the maximum of $\phi_{x}$. The time evolution of the three quantities $\Pi, S$, and $R$ is shown in Fig. 10. Panel (a) [(b)] corresponds to $r_{0}=0\left(r_{0}=5\right)$. For $r_{0}=0$, the momentum $\Pi$ goes through 0 for $R=0$ (collision instant). There $S>0$, so that $\Pi$ will keep decreasing and

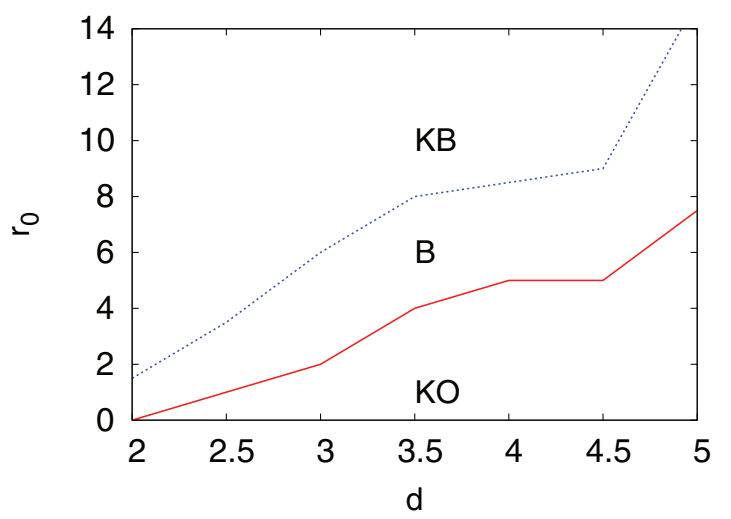

FIG. 16. (Color online) Parameter plane $\left(d, r_{0}\right)$ showing the different possible outcomes for a kink collision, a fast breather (B), a kink-oscillon metastable state $(\mathrm{KO})$, and a kink-breather metastable state (KB).

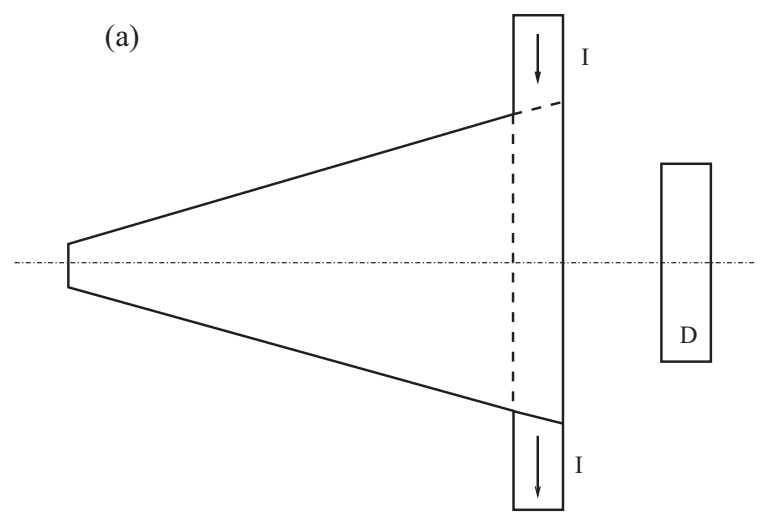

(b)

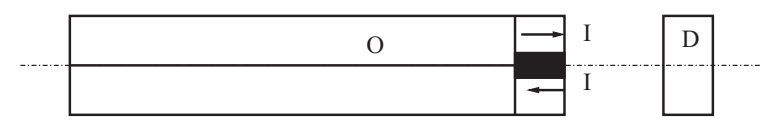

FIG. 17. Sketch of a microwave generator based on a radial Josephson window junction.

oscillate around zero, indicating that the soliton is destroyed. For $r_{0}=5$ shown in the bottom panel, $\Pi>0$ at collision and $S>0$ so that $\Pi$ remains positive for $t \geqslant 20$. After that instant, $S$ starts to oscillate with a period of about 6 . $S$ is largely positive so that $\Pi<0$ on average. We then reach $\Pi=0$ so that the kink stops at $t \approx 41$, for which $R=15$. This is the return effect. The total energy is conserved but gets distributed differently between the different components, the kinetic term $e_{t}=\int_{r_{0}}^{r_{1}} 0.5 \phi_{t}^{2} x d x$, the gradient term $e_{x}=\int_{r_{0}}^{r_{1}} 0.5 x \phi_{x}^{2} d x$, and the potential term $e_{\cos }=\int_{r_{0}}^{r_{1}}(1-\cos \phi) x d x$. Initially the kink has 0 velocity so that $e_{t}=0$; all the energy is concentrated in $e_{x}$ and $e_{p}$. For $r_{0}=0$ in Fig. 11(a), the kinetic term $e_{t}$ increases from 0 to its maximum at the collision and then remains about constant. The potential term $e_{p}$ decreases from its maximum value at $t=0$ and stabilizes around half its value. The behavior is different for $r_{0}=10$ shown in Fig. 11(b), for which the collision is almost elastic. There at the instant of collision, the kinetic energy reaches its maximum, and the total energy, potential energy, and gradient energies are almost zero. After collision, both recover their initial values.

\section{B. Collision in three and higher dimensions}

We now consider the collision of a kink in three and higher dimensions to see if there are particular situations. The situation is qualitatively similar to the $2 \mathrm{D}$ case. Radiation forms as a "bump" as the kink shrinks toward $r_{0}$ and stays with the kink. At collision, this radiation is emitted as fast breathers out of the computational domain. As an example, consider the flux of the energy shown for the 3D case in Fig. 12 for $r_{0}=0,5$, and 10 . Interestingly, the case $r_{0}=5$ is similar to the $2 \mathrm{D}$ case for $r_{0}=0$. The kink is entirely destroyed and its energy is shed away in the form of a fast breather that exits the computational domain. This is shown in the series of snapshots in Fig. 13. The fast breather is clearly seen traveling to the right at time $t=31$. For $r_{0}=0$, there is a kink-breather bound state so that the kink "sheds" a breather at every collision with the boundary and decays. Figure 14 shows the successive 
snapshots of the solution in this case. The solution for $t=31$ is clearly a combination of a kink and a breather. For such a large value of $d$ and such a small $r_{0}$, the radial term $(d-1) \phi_{r} / r$ is very strong and prevents a low-frequency breather from escaping. Only breathers of frequency $\omega \approx 1$ can escape, and these have fairly low energy. Such a breather will be "shed" from the kink as it reaches its return point, around $R=5$. The energy and momentum behave in a very similar way as in the $2 \mathrm{D}$ case, so we do not present them.

To confirm these findings, we conducted two simulations with $d=5$ for $r_{0}=0$ and 5 and $r_{1}=40$. The flux of energy exiting the domain is shown in Fig. 15. It shows two breathers being emitted, respectively, at $t=55$ and 100 for $r_{0}=0$. The $\left(d, r_{0}\right)$ parameter plane is shown in Fig. 16. It shows the coexistence of the three states: the fast breather (B), the kink-breather metastable (KB), and the kink-oscillon metastable state $(\mathrm{KO})$. Interestingly, the last one cannot be seen for $d=2$.

\section{CONCLUSION}

Motivated by theory and applications, we studied radial sine-Gordon kinks in two and higher dimensions. A full twodimensional simulation showed that azimuthal perturbations remain small. We therefore reduced the problem to the onedimensional radial sine-Gordon equation, which we solve on an interval $\left[r_{0}, r_{1}\right]$. Before collision, the kink is well described by a simple law derived from the conservation of energy. In two dimensions, the collision of the kink with the boundary $r_{0}$ will result in a fast breather for small $r_{0}$ and in a kink-breather metastable state for larger $r_{0}$. In the latter, the kink sheds at each "return" a large part of its energy into bursts, which are breather solutions. We have characterized these waves in terms of their energy, frequency, and velocity. In three and higher dimensions and small $r_{0}$, we observe a kink oscillon bound state. The three states exist in the $\left(d, r_{0}\right)$ parameter space. This study shows that radial perturbation opens a channel between the kink solutions and the breather solutions. This is particularly interesting because in one dimension these are completely separated. This additional term provides, therefore, a mechanism to "destroy" these solutions and extract the energy they contain.

In view of applications to 2D Josephson junctions, this could be very useful to generate terahertz radiation. At this time, output from these devices is low. Here for small $r_{0}$ all the kink energy is converted into radiation. This suggests the design of a new device based on window Josephson junctions. A sketch of the device is shown in Fig. 17. Panel (a) shows a top view of the junction together with the radiofrequency detector $D$. Notice that the current input is similar to that in [19] with its passive region separating the electrodes. Panel (b) shows a side view of the system with the oxide layer $\mathrm{O}$ separating the two superconducting films. As the current $I$ is increased, a train of fluxons is formed. These reflect into the narrow end $r=r_{0}$ and fast breathers are formed that consist of bursts of microwaves. Since all the kink energy is converted into microwaves, we expect this system to generate much more radiation than a standard flux-flow.

\section{ACKNOWLEDGMENTS}

The authors thank Egor Alfimov, Peter Christiansen, Yuri Gaididei, and Boris Malomed for very helpful discussions. J.G.C. thanks the Department of Informatics and Mathematical Modelling and the Department of Mathematics at the Technical University of Denmark for their hospitality during several visits. The authors acknowledge the Centre de Resources Informatiques de Haute Normandie, where most of the calculations were done.
[1] R. K. Dodd, J. C. Eilbeck, J. D. Gibbon, and H. C. Morris, Solitons and Nonlinear Wave Equations (Academic Press Inc., London, 1982).

[2] P. L. Christiansen and O. H. Olsen, Phys. Scr. 20, 531 (1979); Phys. Lett. A 68, 185 (1978).

[3] P. S. Lomdahl, O. H. Olsen, and P. L. Christiansen, Phys. Lett. A 78, 125 (1980).

[4] P. L. Christiansen and P. S. Lomdahl, Physica D 2, 482 (1981).

[5] J. Geicke, Physica D 4, 197 (1982).

[6] J. Geicke, Phys. Scr. 29, 431 (1984).

[7] J. Geicke, Phys. Lett. A 98, 147 (1983).

[8] I. L. Bogolubsky and V. G. Makhankov, Pis'ma Zh. Eksp. Teor. Fiz. 24, 15 (1976) [JETP Lett. 24, 12 (1976)].

[9] B. Piette and W. J. Zakrzewski, Nonlinearity 11, 1103 (1998).

[10] M. Gleiser and D. Sicilia, Phys. Rev. D 80, 125037 (2009).

[11] E. M. Maslov, Physica D 15, 433 (1985).

[12] B. A. Malomed, Physica D 24, 155 (1987).

[13] B. A. Malomed and E. M. Maslov, Phys. Lett. A 160, 233 (1991).

[14] G. L. Alfimov, W. A. B. Evans, and L. Vàzquez, Nonlinearity 13, 1657 (2000).

[15] A. Barone and G.-F. Paterno, Physics and Applications of the Josephson Effect (Wiley, New York, 1982).
[16] S. Pagano, C. Nappi, R. Cristiano, E. Esposito, L. Frunzio, L. Parlato, G. Peluso, G. Pepe, and U. Scott Di Uccio, Nonlinear Superconducting Devices and High $T_{c}$ Materials (World Scientific, Singapore, 1995).

[17] A. Benabdallah, J. G. Caputo, and A. C. Scott, Phys. Rev. B 54, 16139 (1996).

[18] A. Benabdallah, J. G. Caputo, and A. C. Scott, J. Appl. Phys. 88, 3527 (2000).

[19] G. Carapella, N. Martucciello, and G. Costabile, Phys. Rev. B 66, 134531 (2002).

[20] M. Jaworski, Phys. Rev. B 71, 214515 (2005).

[21] COMSOL MULTIPHYSICS modeling and simulation software, http://www.comsol.com/.

[22] E. Hairer, S. P. Norsett, and G. Wanner, Solving Ordi-nary Differential Equations I (Springer-Verlag, Berlin, 1987).

[23] B. Leimkhuler and S. Reich, Simulating Hamiltonian Dynamics (Cambridge University Press, Cambridge, 2004).

[24] M. R. Samuelsen, Phys. Lett. A 74, 21 (1979).

[25] M. Abramowitz and I. Stegun, Handbook of Mathematical Functions (Dover, New York, 1972).

[26] A. C. Scott, Nonlinear Science (Oxford University Press, Oxford, 1999).

[27] S. Dutta, D. A. Steer, and T. Vachaspati, Phys. Rev. Lett. 101, 121601 (2008). 